\title{
Blind Joint Soft-Detection Assisted Slow Frequency-Hopping Multicarrier DS-CDMA
}

\author{
Lie-Liang Yang, Member, IEEE, and Lajos Hanzo, Senior Member, IEEE
}

\begin{abstract}
A novel multiple-access scheme based on slow frequency-hopping multicarrier direct-sequence, code-division multiple access (SFH/MC DS-CDMA) is proposed and investigated, which can be rendered compatible with the existing second-generation narrow-band CDMA and third-generation wide-band CDMA systems. Blind joint soft-detection of the SFH/MC DS-CDMA signals is investigated, assuming that the receiver has no knowledge of the associated frequency-hopping (FH) patterns invoked. The system's performance is evaluated over the range of Nakagami multipath fading channels. The results show that blind joint softdetection achieves the required bit-error rate performance, while blindly acquiring the FH patterns employed. This is advantageous during the commencement of communications or during soft handover.
\end{abstract}

Index Terms-Blind detection, code-division multiple access, constant-weight codes, frequency-hopping, orthogonal frequency-division multiplexing.

\section{INTRODUCTION}

$\mathbf{R}$ ECENTLY, a considerable number of studies based on wide-band code-division multiple access (W-CDMA) [1], [2] and multicarrier code-division multiple access (MC-CDMA) [3]-[10] have been conducted in the context of third-generation mobile communication systems. However, broad-band systems with bandwidths much wider than that of the third-generation systems are required for meeting future requirements. Hence, compatibility with both the emerging broad-band access networks (BRAN), which have opted for a multicarrier orthogonal frequency-division multiplexing (OFDM)-based solution and the existing second- and third-generation CDMA systems is an important consideration.

A potential candidate multiple-access scheme meeting these requirements is constituted by frequency-hopping multicarrier direct-sequence CDMA (FH/MC DS-CDMA)[9], [10], where the entire bandwidth of future systems can be divided into a number of subbands and each subband can be assigned a subcarrier. According to the prevalent service requirements, the set of legitimate subcarriers can be distributed in line with the instantaneous information rate requirements. FH techniques are employed for each user, in order to occupy the whole system band-

Paper approved by A. Goldsmith, the Editor for Wireless Communication of the IEEE Communications Society. Manuscript received May 15, 1999; revised January 15, 2000. This work was supported by the EPSRC, Swindon, U.K., and the Commission of the European Communities, Brussels, Belgium. This paper was presented in part at the IEEE GLOBECOM'99, Rio de Janeiro, Brazil, December 5-9, 1999.

The authors are with the Department of Electronics and Computer Science, University of Southampton, SO17 1BJ, UK (e-mail: lh@ecs.soton.ac.uk).

Publisher Item Identifier S 0090-6778(00)07537-1. width and to efficiently utilize the system's frequency resources. Specifically, slow $\mathrm{FH}(\mathrm{SFH})$, fast $\mathrm{FH}$, or adaptive $\mathrm{FH}$ techniques can be utilized depending on the system's design and the state of the art. In FH/MC DS-CDMA systems, the subbands are not required to be of equal bandwidth. Hence, existing second- and third-generation CDMA systems can be supported using one or more subcarriers, consequently simplifying the frequency resource management and efficiently utilizing the entire bandwidth available. This regime can also remove the spectrum segmentation of existing "legacy" systems, while ensuring compatibility with future BRAN and unlicensed systems. Furthermore, a number of subchannels with variable processing gains can be employed - a technique that was developed for third-generation mobile systems - in order to support various services requiring low- to very high-rate transmissions, for example, for wireless Internet access.

In this contribution, we consider the above $\mathrm{FH} / \mathrm{MC}$ DS-CDMA system, when using SFH and BPSK data modulation. The system operates in a multipath fading environment, and a RAKE receiver structure with maximum-ratio combining (MRC) is used for demodulation. We assume that the receiver has no knowledge of the FH patterns employed. Hence, blind joint soft-detection using maximum-likelihood sequence detection (MLSD) - which is considered to be the optimum scheme-can be utilized, in order to simultaneously accomplish both information detection and $\mathrm{FH}$ pattern acquisition. The system performance is evaluated over the range of Nakagami- $m$ multipath fading channels, since they describe the urban multipath channel [11], and its probability density function (pdf) models a continuous transition from a Rayleigh fading channel to a nonfading Gaussian channel by varying a single parameter, namely $m$, from one to infinity [11]-[16]. Furthermore, the Nakagami- $m$ distribution offers features of analytical convenience, which makes it possible to evaluate the system performance by using both analytical and numerical approaches.

The reminder of this paper is organized as follows. In Section II, we discuss the proposed SFH/MC DS-CDMA system, which includes the description of the transmitted signal, the channel model, and the receiver model. In Section III, we develop the above-mentioned blind joint soft-detection scheme for our SFH/MC DS-CDMA system for the case when the receiver is oblivious of the FH patterns used, while in Section IV, we investigate the system's performance using symbol-by-symbol MLSD. The numerical results and comparisons are given in Section V, and finally, our conclusions are offered in Section VI. Let us now consider the proposed system. 


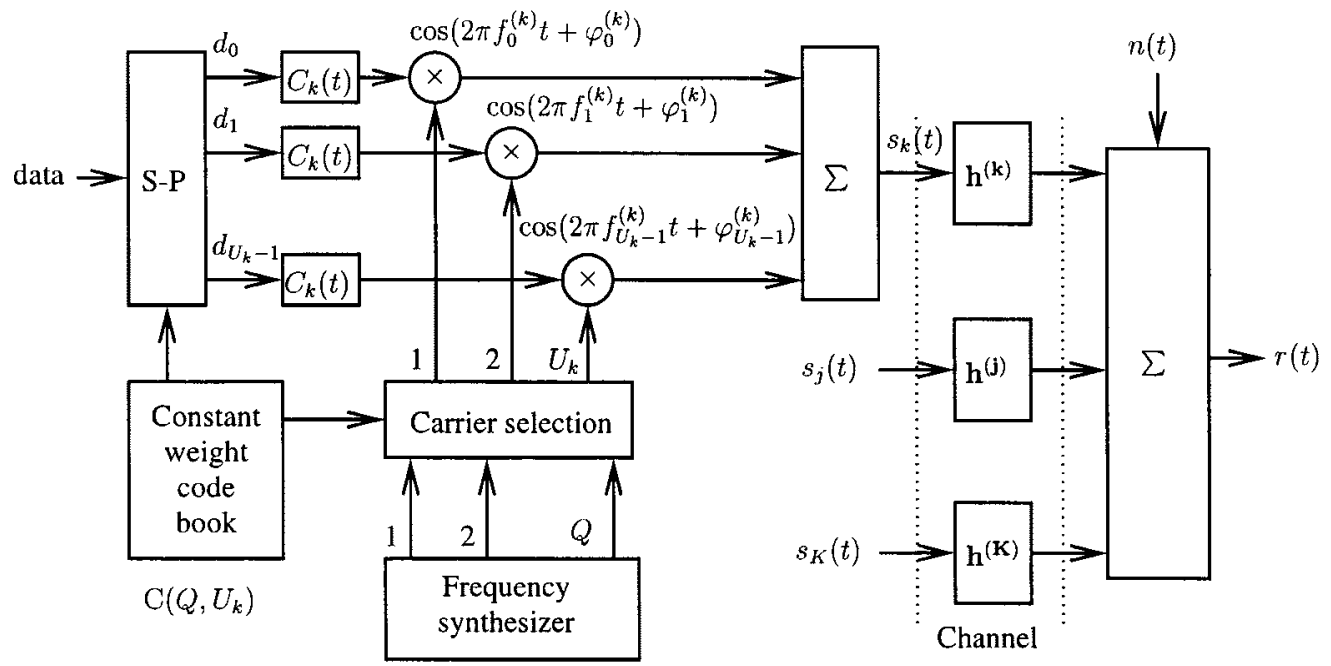

Fig. 1. Transmitter and channel block diagram of the FH/MC DS-CDMA system.

\section{SFH/MC DS-CDMA SYSTEM}

\section{A. Transmitted Signal}

The model of the transmitter and the multiple-access channel is depicted in Fig. 1. Each of the $K$ users in the system is assigned a randomly generated signature sequence, which produce spread, wide-band signals. In the figure, $\mathrm{C}\left(Q, U_{k}\right)$ represents a constant-weight code (CWC) of user $k$ with $U_{k}$ number of "1"s and $\left(Q-U_{k}\right)$ number of " 0 "s, i.e., the weight of $\mathrm{C}\left(Q, U_{k}\right)$ is $U_{k}$. This code is read from a so-called CWC book, which represents the $\mathrm{FH}$ patterns. Theoretically, the size of the CWC book is $\left(\begin{array}{c}Q \\ U_{k}\end{array}\right)=Q ! / U_{k} !\left(Q-U_{k}\right) !$. The CWC $\mathrm{C}\left(Q, U_{k}\right)$ plays two different roles. Its first role is that its weight-namely $U_{k}$-determines the number of subcarriers involved, while its second function is that the positions of the $U_{k}$ number of binary " 1 "s determines the selection of a set of $U_{k}$ number of subcarrier frequencies from the $Q$ number of outputs of the frequency synthesizer.

At the transmitter of the $k$ th user in Fig. 1, the bit stream having a bit duration of $T_{b}$ is first serial-to-parallel (S-P) converted, yielding $U_{k}$ parallel streams, which is controlled by the CWC C $\left(Q, U_{k}\right)$. Let the new bit duration of each parallel stream be expressed as $T$, which can be $T=U_{k} T_{b}$ or $T=T_{b}$, depending on the objectives of the system design, as it will be augmented during our further discourse. For example, if the design aims to mitigate the intersymbol interference (ISI) in a high bit-rate transmission scheme, a high bit duration is required, and hence, $T=U_{k} T_{b}$ can be employed. However, if the design aims to support multiple information rates, a constant bit duration can be employed, and multirate transmissions are implemented by employing a different number of subcarriers. Theoretically, $Q$ number of different information rates can be supported by changing the weight of the code $\mathrm{C}\left(Q, U_{k}\right)$. As seen in Fig. 1, after S-P conversion, each stream is direct-sequence (DS) spread, in order to form the spread wide-band signal, and this spread signal then modulates one of the selected subcarriers. Finally, the transmitted signal of the $k$ th user can be expressed as

$$
s_{k}(t)=\sum_{u_{k}=0}^{U_{k}-1} \sqrt{2 P} d_{u_{k}}^{(k)}(t) c_{k}(t) \cos \left(2 \pi f_{u_{k}}^{(k)} t+\varphi_{u_{k}}^{(k)}\right)
$$

where $P$ represents the transmitted power per carrier, while $U_{k}$ indicates the weight of the CWC currently employed by the $k$ th user. Furthermore, $\left\{d_{u_{k}}^{(k)}(t)\right\},\left\{c_{k}(t)\right\},\left\{f_{u_{k}}^{(k)}\right\}$, and $\left\{\varphi_{u_{k}}^{(k)}\right\}$ represent the current data stream's waveforms, the DS spreading waveforms, the subcarrier frequency set, and the phase angles introduced in the carrier modulation process. The data stream's waveform $d_{u_{k}}^{(k)}(t)=\sum_{i=-\infty}^{\infty} d_{u_{k}}^{(k)} P_{T}(t-i T)$ consists of a sequence of mutually independent rectangular pulses of duration $T$ and of amplitude +1 or -1 with equal probability. The spreading sequence $c_{k}(t)=\sum_{j=-\infty}^{\infty} c_{j}^{(k)} \Pi\left(t-j T_{c}\right)$ denotes the signature sequence waveform of the $k$ th user, where $c_{j}^{(k)}$ assumes values of +1 or -1 with equal probability, while $\Pi(t)$ is the common chip waveform for all signals. The chip waveform is time limited or bandwidth limited and it is also normalized according to $\int_{0}^{T_{c}} \Pi^{2}(t) d t=T_{c}$. In this paper-for the sake of simplicity-we assume that there exists no spectral overlap between the spectral main-lobes of two adjacent subcarriers after the spreading [6], and also assume that there exists no interference between subcarriers-i.e., interference is inflicted only, when an interfering user activates the same subcarrier, as the reference user. Readers interested in the effect of overlapping subcarriers are referred to, for example, [3] and [7]. Let $N=T_{b} / T_{c}$, then the processing gain of $N_{P}=T / T_{c}$ equals to $U_{k} N$ or $N$ depending on the system's objectives, as discussed previously, i.e., whether $T=U_{k} T_{b}$ or $T=T_{b}$. Furthermore, we assume that the FH duration is $T_{h}$, and that the number of data bits $N_{b}=T_{h} / T$ transmitted per hop is a positive integer, which is strictly larger than 1, i.e., we assume using SFH.

\section{B. Channel Description}

The channel model considered in this paper is the commonly used finite-length tapped delay line model of a frequency-selective multipath channel, whose complex low-pass impulse response for subcarrier $u_{k}$ of user $k$ is given by [19, p. 796, eq. (14-5-8)]:

$$
h_{u_{k}}^{(k)}(t)=\sum_{l_{p}=0}^{L_{p}-1} \alpha_{u_{k}, l_{p}}^{(k)} e^{j \phi_{u_{k}, l_{p}}^{(k)}} \delta\left(t-l_{p} T_{c}\right)
$$




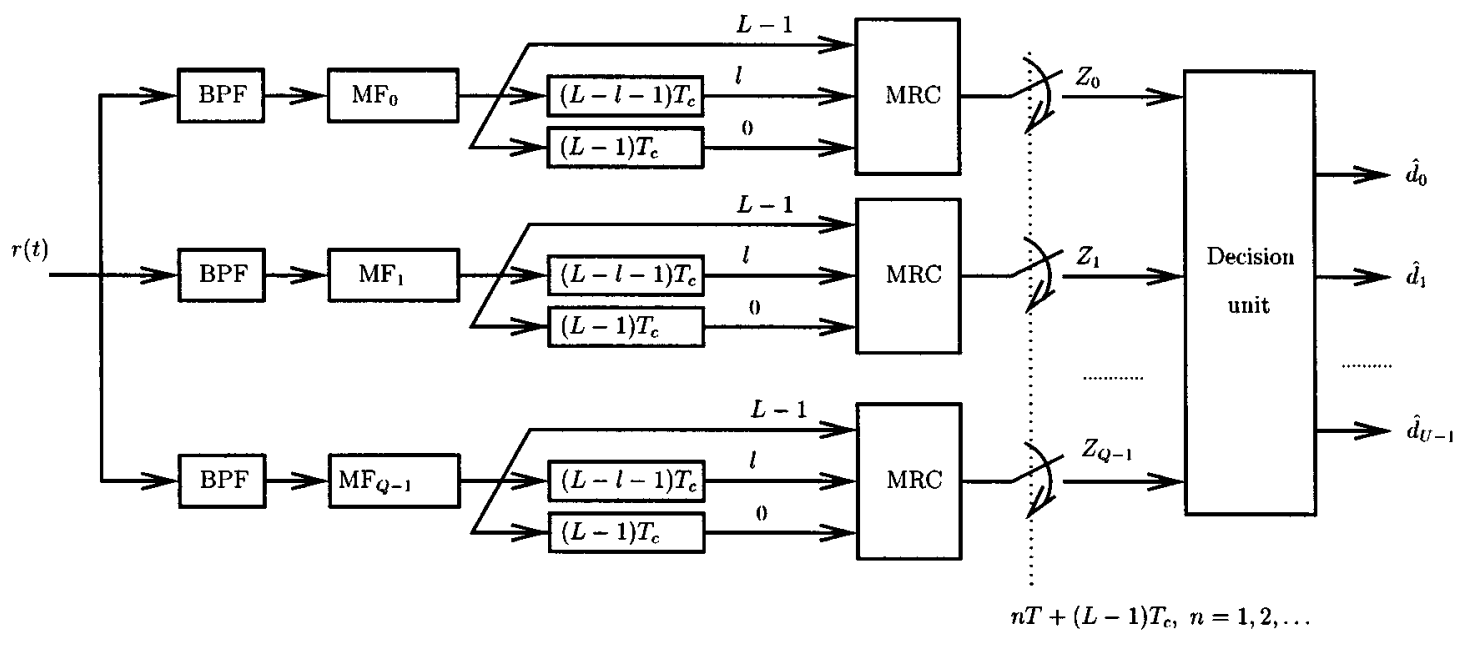

Fig. 2. Receiver block diagram of the FH/MC DS-CDMA system.

where $l_{p} T_{c}$ is the relative delay of the $l_{p}$ th path of user $k$ with respect to the main path, the phases $\left\{\phi_{u_{k},}^{(k)}, l_{p}\right\}$ are independent identically distributed (i.i.d.) random variables uniformly distributed in the interval $(0,2 \pi)$, whilst the $L_{p}$ tap weights $\left\{\alpha_{u_{k}, l_{p}}^{(k)}\right\}$ are independent Nakagami random variables with a pdf of [13]

$$
\begin{aligned}
p\left(\alpha_{u_{k}, l_{p}}^{(k)}\right) & =M\left(\alpha_{u_{k}, l_{p}}^{(k)}, m, \Omega_{u_{k}, l_{p}}^{(k)}\right) \\
M(R, m, \Omega) & =\frac{2 m^{m} R^{2 m-1}}{\Gamma(m) \Omega^{m}} e^{(-m / \Omega) R^{2}}
\end{aligned}
$$

where $\Gamma(\cdot)$ is the gamma function, and $m$ is the Nakagami- $m$ fading parameter, which is equal to

$$
m=E^{2}\left[\left(\alpha_{u_{k}, l_{p}}^{(k)}\right)^{2}\right] / \operatorname{var}\left[\left(\alpha_{u_{k}, l_{p}}^{(k)}\right)^{2}\right] .
$$

The parameter $m$ of the amplitude distribution characterizes the severity of the fading over the $l_{p}$ th resolvable path [11]. It is well known that $m=1$ corresponds to Rayleigh fading, $m \rightarrow \infty$ corresponds to the nonfading condition, and $m=1 / 2$ corresponds to the so-called one-side Gaussian fading, i.e., the worst-case fading condition. The Rician and lognormal distributions can also be closely approximated by the Nakagami distribution with $m>1$. For more detailed information concerning the Nakagami distribution, readers are referred to the excellent overview by Simon and Alouini [12]. The parameter $\Omega_{u_{k}, l_{p}}^{(k)}$ in (3) is the second moment of $\alpha_{u_{k}}^{(k)}, l_{p}$, i.e., $\Omega_{u_{k}, l_{p}}^{(k)}=E\left[\left(\alpha_{u_{k}, l_{p}}^{(k)}\right)^{2}\right]$. We assume a negative exponentially decaying multipath intensity profile (MIP) distribution given by

$$
\Omega_{u_{k}, l_{p}}^{(k)}=\Omega_{u_{k}, 0}^{(k)} e^{-\eta l_{p}}, \quad \eta \geq 0
$$

where $\Omega_{u_{k}, 0}^{(k)}$ is the average signal strength corresponding to the first resolvable path and $\eta$ is the rate of average power decay. Consequently, for an asynchronous CDMA system with $K$ users, the received signal takes the form

$$
\begin{array}{r}
r(t)=n(t)+\sqrt{2 P} \sum_{k=1}^{K} \sum_{u_{k}=0}^{U_{k}-1} \sum_{l_{p}=0}^{L_{p}-1} \alpha_{u_{k}, l_{p}}^{(k)} d_{u_{k}}^{(k)}\left(t-l_{p} T_{c}-\tau_{k}\right) \\
\cdot c_{k}\left(t-l_{p} T_{c}-\tau_{k}\right) \cos \left(2 \pi f_{u_{k}}^{(k)} t+\psi_{u_{k}}^{(k)}, l_{p}\right)
\end{array}
$$

where $n(t)$ represents the additive white Gaussian noise (AWGN) with zero mean and double-sided power spectral density of $N_{0} / 2, \psi_{u_{k}, l_{p}}^{(k)}=\left[\varphi_{u_{k}}^{(k)}+\phi_{u_{k}, l_{p}}^{(k)}-2 \pi f_{u_{k}}^{(k)}\left(\tau_{k}+l_{p} T_{c}\right)\right]$ $(\bmod 2 \pi)$, which is assumed to be an i.i.d. random variable having a uniform distribution in $(0,2 \pi)$, and $\tau_{k}$ represents the propagation delay of user $k$.

\section{Receiver Model}

Let the first user be the user of interest and consider the conventional matched filter-based RAKE receiver with MRC, as shown in Fig. 2, where the superscript and subscript of the reference user's signal has been omitted for notational convenience. In Fig. $2, L, 1 \leq L \leq L_{p}$-the number of diversity branches used by the receiver-is a variable, allowing us to study the effect of different diversity orders. In contrast to the transmitter side, where only $U_{k}$ out of $Q$ subcarriers are transmitted by the user $k$, at the receiver all $Q$ subcarriers are always tentatively demodulated. The information bits transmitted over the different subcarriers are blind soft-detected jointly using the MLSD approach. Consequently, from the point of view of the receiver, each subcarrier can be viewed as an on-off type signaling scheme. When a subcarrier is actively used for signaling and hence it is in the on-state, the MRC output samples give +1 or -1 information, otherwise, while passive and hence in the off-state, the MRC stage outputs noise. Let us now discuss the proposed blind joint soft-detection.

\section{BLIND JoINT SOFT-DETECTION OF SFH/MC DS-CDMA SIGNALS}

Since the receiver is not equipped with the explicit knowledge of the FH patterns, at the commencement of communications, or in case of soft handovers to other base stations, the SFH/MC DS-CDMA signals can only be detected blindly. Hence, in this section, we invoke the blind joint soft-detection for the SFH/MC DS-CDMA signals by first considering the statistics of the MRC output samples upon invoking the often-used Gaussian approximation. 


\section{A. Statistics of the MRC Output Samples: Gaussian Approximation}

The $Q$ number of matched filters in the receiver of Fig. 2 are matched to the reference user's CDMA code used for spreading in the subbands associated with the $Q$ number of subcarriers, and are assumed to have achieved time synchronization with the corresponding initial path of the subcarriers of the reference signal. If we assume that perfect estimates of the channel tap weights are available, then after appropriately delaying the individual matched filter outputs, in order to synchronize the $L$ number of path signals used by the RAKE combiner, the $q$ th MRC output sampled at $t=n T+(L-1) T_{c}$, in order to detect the $n$th symbol can be expressed as

$$
Z_{q}[n]=D_{q}[n]+I_{q}[n]
$$

where $D_{q}[n]$ and $I_{q}[n]$ represent the desire component and the interference plus noise component, respectively.

Assuming that $Z_{q}[n]$ is an independent Gaussian distributed variable-i.e., Gaussian approximation is imposed on the multipath and multiple-access interference-then the mean value of $Z_{q}[n]$ can be expressed as [13], [15]

$$
D_{q}[n]=\sqrt{\frac{P}{2}} T d_{q}(n) \sum_{l=0}^{L-1} \alpha_{q, l}^{2}
$$

where $d_{q}(n)$ is the $n$th bit transmitted on subcarrier $q$ by the reference user and $d_{q}(n) \in\{+1,-1,0\}$ with " 0 " representing the off-state. As we discussed previously, we assume that there exists no spectrum overlap between the spectral main-lobes of two adjacent subcarriers [6], and interference is inflicted only, when an interfering user activates the same subcarrier, as the reference user, which is referred as the so-called hit [17]. Consequently, assuming that there exists $K_{h}\left(0 \leq K_{h} \leq K-1\right)$ number of interfering signals, all of which activate the $q$ th subcarrier during the $n$th symbol of the reference signal, then the variance of $Z_{q}[n]$ can be expressed as [13], [15]

$$
\begin{aligned}
\sigma^{2}=\frac{P T^{2}}{2}\left[\frac{q\left(L_{p}, \eta\right)-1}{2 N_{p}}+\frac{K_{h} q\left(L_{p}, \eta\right)}{3 N_{p}}+\right. & \left.\left(\frac{2 \Omega_{0} E_{b}}{N_{0}}\right)^{-1}\right] \\
& \cdot \Omega_{0} \sum_{l=0}^{L-1} \alpha_{q, l}^{2}
\end{aligned}
$$

where $E_{b}=P T$ is the average transmitted energy-per-bit, $q\left(L_{p}, \eta\right)=\left(1-e^{-\eta L_{p}}\right) /\left(1-e^{-\eta}\right)$, if $\eta \neq 0$, otherwise, $q\left(L_{p}, \eta\right)=L_{p}$, if $\eta=0$. Hence, the pdf of the $q$ th sampled subchannel output in Fig. 2 can be expressed as

$$
p_{q}(z)=\frac{1}{\sqrt{2 \pi} \sigma} \exp \left(-\frac{\left(z-D_{q}[n]\right)^{2}}{2 \sigma^{2}}\right) .
$$

\section{B. $M L S D$}

If we assume that the transmitted signals are equally probable, symbol-by-symbol MLSD is considered to be the optimum detection scheme [19], [20]. In this section, we investigate the MLSD of the SFH/MC DS-CDMA signals under the assumption that all symbols (vectors) are transmitted with equal probability and that the MRC output samples are mutually independent random variables having common pdfs given by (9). Moreover, we assume that $\alpha_{q, l}=\alpha_{l}$ for $q=0,1, \ldots, Q-1$
Let assume that the receiver only knows the weight of the transmitted CWC, but not the positions of the binary " 1 "s; hence, the receiver has to detect not only the positions of the "1"s, which indicate the subcarriers used, but also the antipodal binary modulated information conveyed by the activated subcarriers. Let us express the input data in the vectorial form of $\mathbf{D}_{i}=\left\{d_{i, 0}, d_{i, 1}, \ldots, d_{i, Q-1}\right\}$, where $0 \leq i \leq M-1$, and $M$ is the number of different vectors representing the possible transmitted symbols, which can be expressed as $M=\left(\begin{array}{c}Q \\ U\end{array}\right) 2^{U}$ for the CWC C $(Q, U)$ involved. Then for a set of $Q$ MRC samples $\mathbf{Z}=\left\{Z_{1}, Z_{2}, \ldots, Z_{Q-1}\right\}$ in Fig. 2-which we refer to here as a received symbol or vector-the MLSD blind joint soft-detection is based on finding the data vector $\mathbf{D}_{i}$ maximizing the probability [20]

$$
\begin{aligned}
p\left(\mathbf{Z} \mid \mathbf{D}_{i}\right)= & \frac{1}{\left(2 \pi \sigma^{2}\right)^{Q / 2}} \\
& \cdot \exp \left(-\frac{\left.\sum_{q=0}^{Q-1}\left(Z_{q}-\sqrt{\frac{P}{2}} T d_{i, q} \sum_{l=0}^{L-1} \alpha_{l}^{2}\right)^{2}\right)}{2 \sigma^{2}}\right), \\
& (i=0,1, \ldots, M-1 .
\end{aligned}
$$

Here, we define

$$
\begin{aligned}
D\left(\mathbf{Z}, \mathbf{D}_{i}\right)= & \sum_{q=0}^{Q-1}\left|Z_{q}-\sqrt{\frac{P}{2}} T d_{i, q} \sum_{l=0}^{L-1} \alpha_{l}^{2}\right|^{2} \\
= & \sum_{q=0}^{Q-1} Z_{q}^{2}-2\left(\sqrt{\frac{P}{2}} T \sum_{l=0}^{L-1} \alpha_{l}^{2}\right) \sum_{q=0}^{Q-1} Z_{q} d_{i, q} \\
& +\left(\sqrt{\frac{P}{2}} T \sum_{l=0}^{L-1} \alpha_{l}^{2}\right)^{2} \sum_{q=0}^{Q-1} d_{i, q}^{2}
\end{aligned}
$$

as the Euclidean distance between the decision variable vector of $\mathbf{Z}$ and the possible transmitted data vector of $\mathbf{D}_{i}$. In (11), the first term on the right-hand side is common for all $i$ values. Furthermore, since vectors from the set $\left\{\mathbf{D}_{i}\right\}$ are derived from the CWC codewords having the same weights, the third term is also common to all computations. Consequently, the MLSD decision criterion based on selecting the signal corresponding to the maximum of the set of probabilities $\left\{P\left(\mathbf{Z} \mid \mathbf{D}_{i}\right)\right\}$ is equivalent to finding the vector $\mathbf{D}_{i}$ that minimizes the Euclidean distance of $D\left(\mathbf{Z}, \mathbf{D}_{i}\right)$, and is in turn equivalent to the maximization of the correlation metrics of

$$
C\left(\mathbf{Z}, \mathbf{D}_{i}\right)=\sum_{q=0}^{Q-1} Z_{q} d_{i, q}, \quad i=0,1, \ldots, M-1 .
$$

The complexity of the detection of a received symbol or vector is determined by both the length and the weight of the CWC. For a CWC $\mathrm{C}(Q, U)$, the detection complexity is proportional to $O\left(\left(\begin{array}{l}Q \\ U\end{array}\right) 2^{U}\right)$-where $O$ indicates the "order" of complexity - since each activated subcarrier position related to a binary "1" in the $\operatorname{CWC~} \mathrm{C}(Q, U)$ can be associated with a +1 or -1 data bit. However, if $Q$ different rate transmission schemes are assumed, and each rate is invoked with an equal a priori probability, then the average detection complexity can be expressed as $O\left(3^{Q} / Q\right)$. Apparently, this complexity 
is excessive, rendering the associated detection complexity impractically high, when evaluating (12) for all possible code words, if the value of $Q$ is high. Hence, some limitations are required, in order to simplify the detection.

\section{Approach I}

To this effect, let us assume that the synthesizer of Fig. 1 generates a total of $Q=2^{m}$ subcarriers, which coincides with the practical implementational constraints of invoking the fast Fourier transform (FFT) for modulation. Assume furthermore that $U=2^{n}, n=0,1, \ldots, m$ number of subcarriers are activated. Then, the $Q$ number of subcarriers can be divided into $U$ groups, each group having $W=Q / U=2^{m-n}$ subcarriers. A $U$-bit symbol or vector now can be transmitted by $U$ number of subcarriers randomly selected from the $U$ groups, where each group contributes one activated subcarrier. It has been argued by Jung et al.that this arrangement can enhance the so-called "frequency engineering capability" of the system [8]. Under the above constraints, each of the $U$ bits can be detected separately by simply considering the $W$ number of MRC output samples in the same group. Consequently, the detection complexity of the $U$-bit symbol is now proportional to $O(2 U W) \equiv O(2 Q)$, which is linearly dependent on the total number of subcarriers, but independent of the information rate.

Although the complexity of the above detection approach is low, it results in a reduced detection performance. In order to enhance the detection performance and at the same time simplify the computations, the FH patterns can be designed by selecting a subset of codes having a minimum distance of $d$ from the CWCs $\mathrm{C}(Q, U)$, which is now discussed in the next section.

\section{Approach II}

Let $\mathrm{C}(Q, d, U)$ represent a $\mathrm{CWC}$ set having a code length of $Q$ and weight of $U$, as discussed previously. Furthermore, let the minimum distance between any pair of codes from $\mathrm{C}(Q, d, U)$ be $d$. Then, this code constitutes a specific subset of the CWC $\mathrm{C}(Q, U)$, where the number of codewords was $\left(\begin{array}{l}Q \\ U\end{array}\right)$. By contrast, let $\mathrm{A}(Q, d, U)$ represent the number of codewords of the CWC $\mathrm{C}(Q, d, U)$. Then, if the $\mathrm{FH}$ patterns are determined now by all the $\mathrm{A}(Q, d, U)$ codewords, the detection complexity will be reduced from $O\left(\left(\begin{array}{l}Q \\ C\end{array}\right) 2^{U}\right)$ to $O\left(\mathrm{~A}(Q, d, U) 2^{U}\right)$.

The nonlinear $\operatorname{CWC~C}(Q, d, U)$, where $d=2 v$ with $v$ being a positive integer, has some well-known properties. The CWCs constitute a class of efficient codes suitable for error correction or error detection over both binary symmetrical and asymmetrical channels [22]. Due to space limitations, the interested reader is referred to [21] and [22, p. 528] for a discussion on the more detailed properties of CWCs, especially for notes on the bounding property of $\mathrm{A}(Q, d, U)$ related to our analysis.

\section{Performance of THE SFH/MC DS-CDMA System EMPLOYING BLIND JOINT SOFT-DETECTION}

As shown for example in Fig. 3, for the SFH/MC DS-CDMA system using a $\mathrm{CWC} \mathrm{C}(Q, 2 v, U)$ in order to activate the subcarriers and using a receiver without the explicit knowledge of the FH patterns, but knowing the number of the active subcar-

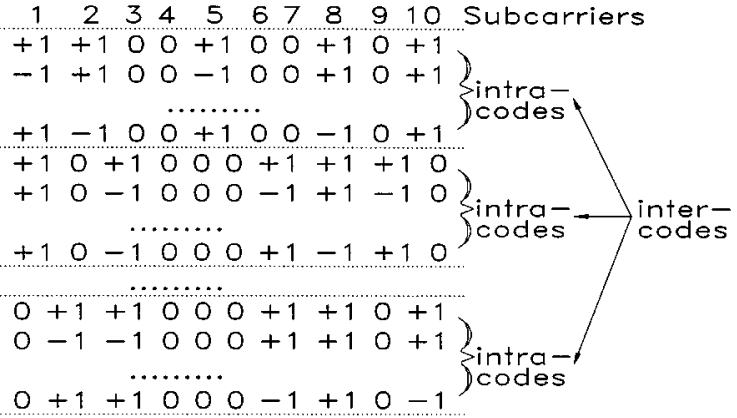

Fig. 3. Example of intracodes and intercodes of a $\mathrm{CWC} \mathrm{C}(10,4,5)$ for SFH/MC DS-CDMA system using ten subcarriers.

riers, the receiver has to evaluate the previously derived correlation metrics. These metrics must be evaluated for two different scenarios in order to demodulate a parallel $U$-bit symbol or vector.

Specifically, we have to consider those codes, which have identical "1" positions in the FH code, corresponding to identical activated subcarriers, but potentially conveying different data symbols on these active subcarriers. We refer to these as intraset codes or intracodes, for short. Explicitly, the above intracodes are derived from the same CWC and activate the same subcarriers. By contrast, the so-called intercodes are derived from different CWCs having the same weights, but activating different subcarriers.

Consequently, in our proposed blind joint soft-detection scheme, two different types of errors exist, the intracode errors and the intercode errors, the probabilities of which are denoted by $P_{\text {intra }}(\cdot)$ and $P_{\text {inter }}(\cdot)$, respectively. The intracode errors do not result in opting for a code other than the transmitter's code, they simply result in some bits being demodulated erroneously. By contrast, the intercode errors may lead not only to erroneous bit decisions, but also to opting for another CWC, hence potentially yielding more severe decision errors. Note that, since the intracode errors are resulted from the same $\mathrm{CWC}$, the intracode error probability is, in fact, equivalent to the error probability of the SFH/MC DS-CDMA system with its receiver having the explicit knowledge of the $\mathrm{FH}$ patterns and using soft-detection. Since the derivation of the exact expression for the probability of correct vector or symbol decoding is complicated by the correlations amongst all the $A(Q, 2 v, U) 2^{U}$ number of correlation metrics, the union upper bound (UUB) probability of error can be invoked.

Let the entire set of $A(Q, 2 v, U) 2^{U}$ number of information vectors or symbols be divided into $A(Q, 2 v, U)$ number of sets, where the vectors from the same set activate the same subcarriers, and hence, the vectors of the same set belong to intracodes. Logically, the vectors of different sets constitute intercodes. Let the first vector in the first set, which is expressed as $X_{11}$, be transmitted. Then, the UUB probability of an intracode error-which is in fact the UUB probability of a soft-detection assisted SFH/MC DS-CDMA system having the explicit knowledge of the FH patterns - can be expressed as [19]

$$
P_{\text {intra }}(U p)=\sum_{j=2}^{2^{U}} Q\left(\sqrt{\frac{\left|X_{11}-X_{1 j}\right|^{2}}{4 \sigma_{N}^{2}}}\right)
$$


where $X_{1 j}, j \neq 1$ is the $j$ th vector of the first set, $\left|X_{11}-X_{1 j}\right|^{2}$ represents the distance between $X_{11}$ and $X_{1 j}, \sigma_{N}^{2}$ is the normalized variance of the MRC output samples, which is derived by dividing (8) by $\left(\sqrt{P / 2} T \sum_{l=0}^{L-1} \alpha_{l}^{2}\right)^{2}$. By contrast, the UUB probability of an intercode error can be written as

$$
P_{\text {inter }}(U p)=\sum_{i=2}^{A(Q, 2 v, U)} \sum_{j=1}^{2^{U}} Q\left(\sqrt{\frac{\left|X_{11}-X_{i j}\right|^{2}}{4 \sigma_{N}^{2}}}\right)
$$

where, again, $\left|X_{11}-X_{i j}\right|^{2}$ with $i \neq 1$ represents the distance between $X_{11}$ and $X_{i j}$.

Consequently, the UUB probability of error is the sum of (13) and (14), yielding

$$
P(U p)=P_{\text {intra }}(U p)+P_{\text {inter }}(U p) .
$$

Furthermore, the joint probability of a correct detection plus that of an intracode decision error represents the probability of the event that the receiver selected the correct CWC matched to the transmitted one, corresponding to the acquisition success (AS) probability. With the aid of (13)-(15), we can now approximate the UUB performance of the proposed scheme.

\section{A. Approach I}

Recall from our previous discussions that according to our Approach I, each group of $W$ subcarriers can be separately treated and the codes utilized by the $W$ subcarriers are equivalent to a class of $C(W, 2,1)$ code with $A(W, 2,1)=W$. Furthermore, this class of CWCs, in fact, includes $W$ number of orthogonal intercodes, each activating a single carrier in one of the $W$ possible positions. Since the cross correlation between two intracodes is -1 , the UUB of the intracode error probability conditioned on the tap attenuations can be expressed using (13) as

$$
P_{\text {intra }}(U p \mid S)=Q(\sqrt{\bar{\gamma} S})
$$

where $Q(\cdot)$ is the $Q$-function defined as $Q(x)=$ $(1 / \sqrt{2 \pi}) \int_{x}^{\infty} e^{-t^{2} / 2} d t$ [19] and $\bar{\gamma}$ represents the average signal-to-noise ratio (SNR) for a certain $K_{h}$, which is given by

$$
\bar{\gamma}=\left[\frac{q\left(L_{p}, \eta\right)-1}{2 N_{p}}+\frac{K_{h} q\left(L_{p}, \eta\right)}{3 N_{p}}+\left(\frac{2 \Omega_{0} E_{b}}{N_{0}}\right)^{-1}\right]^{-1}
$$

while $S$-the sum of squares of Nakagami distributed variables describing the multipath components' attenuations-is expressed as

$$
S=\frac{1}{\Omega_{0}} \sum_{l=0}^{L-1} \alpha_{l}^{2} .
$$

The average UUB probability of intracode errors for a given $K_{h}$ is calculated from the conditional upper bound upon weighting $P_{\text {intra }}(U p \mid S)$ by the pdf of $S, P(S)$, and then averaging or integrating the weighted product over its legitimate range. Since $S$ is a sum of squares of Nakagami distributed variables with densities $M\left(\alpha_{l}, m, e^{-\eta l}\right)$, as seen in (3), $S$ can be approximated [11] as the square of another Nakagami random variable, whose density is given by

$$
P(\sqrt{S})=M\left(\sqrt{S}, m_{s}, \Omega_{s}\right)
$$

where $m_{s}=m\left(q(L, \eta)^{2} / q(L, 2 \eta)\right.$ and $\Omega_{s}=q(L, \eta)$. Consequently, upon using (19), the average UUB of the intracode error probability $P_{\text {intra }}(U p)$ for a given $K_{h}$ can be written as

$$
\begin{aligned}
P_{\text {intra }}(U p) & =P\left(K_{h}, \gamma_{s}\right) \\
& =\int_{0}^{\infty} P_{\text {intra }}(U p \mid S) P(\sqrt{S}) d \sqrt{S} \\
& =\int_{0}^{\infty} Q(\sqrt{\bar{\gamma} S}) M\left(\sqrt{S}, m_{s}, \Omega_{s}\right) d \sqrt{S} .
\end{aligned}
$$

The result of the integral of (20) has been given in [13], which was expressed as

$$
\begin{aligned}
P\left(K_{h}, \gamma_{s}\right)= & \sqrt{\frac{\gamma_{s}}{1+\gamma_{s}}} \frac{\left(1+\gamma_{s}\right)^{-m_{s}} \Gamma\left(m_{s}+\frac{1}{2}\right)}{2 \sqrt{\pi} \Gamma\left(m_{s}+1\right)} \\
& \cdot{ }_{2} F_{1}\left(1, m_{s}+\frac{1}{2} ; m_{s}+1 ; \frac{1}{1+\gamma_{s}}\right)
\end{aligned}
$$

where $\gamma_{s}=\left(\bar{\gamma} \Omega_{s} / 2 m_{s}\right)=(\bar{\gamma} q(L, 2 \eta) / 2 m q(L, \eta))$ is the effective SNR per path, ${ }_{2} F_{1}(a, b, ; c ; z)$ is the hypergeometric function defined as [13] ${ }_{2} F_{1}(a, b, ; c ; z)=$ $\sum_{k=0}^{\infty}\left((a)_{k}(b)_{k} z^{k} /(c)_{k} k !\right)$, where $(a)_{k}=a(a+1) \cdots(a+$ $k-1),(a)_{0}=1$.

By contrast, the UUB of an intercode error for a given $K_{h}$ and conditioned on the tap attenuations can be written as

$$
P_{\text {inter }}(U p \mid S)=2(W-1) Q\left(\sqrt{\frac{\bar{\gamma} S}{2}}\right) .
$$

Similarly, the average UUB of the intercode error can be derived, following the approach of the average UUB of the intracode error computation, yielding

$$
P_{\text {inter }}(U p)=2(W-1) P\left(K_{h}, \gamma_{s} / 2\right)
$$

where $P\left(K_{h}, x\right)$ is given by (21). Hence, the UUB of the error probability per bit for a given number of hits $K_{h}$ is the sum of (20) and (23), which is given by

$$
P^{I}\left(K_{h}, \gamma_{s}\right)=P\left(K_{h}, \gamma_{s}\right)+2(W-1) P\left(K_{h}, \gamma_{s} / 2\right) \text {. }
$$

Finally, the average UUB of bit-error probability (BER) can be expressed as [17], [18]

$$
P_{b}=\sum_{K_{h}=0}^{K-1}\left(\begin{array}{c}
K-1 \\
K_{h}
\end{array}\right) P_{h}^{K_{h}}\left(1-P_{h}\right)^{K-1-K_{h}} P^{I}\left(K_{h}, \gamma_{s}\right)
$$

where $0 \leq K_{h} \leq K-1$ and $P^{I}\left(K_{h}, \gamma_{s}\right)$ is given by (24) and $P_{h}$ is the probability of a hit-as defined previously-from an interfering signal. In an asynchronous system, $P_{h}$ can be approximated as

$$
P_{h} \approx \frac{\bar{U}}{Q}
$$

where $\bar{U}$ is the average weights of the constant-weight codewords used in the system.

\section{B. Approach II}

For the previously introduced blind joint soft-decision Approach II, the computation of the UUBs for the probability of intracode and intercode detection errors according to (13) and (14), and the computation of the UUB of the detection error probability according to (15), require the knowledge of the distance between any pairs of CWC codewords. For special sets of CWCs exhibiting an equal distance in the context of arbitrary 
pairs, the exact UUB can be computed by the above-mentioned equations.

Note that for intracodes and for sufficiently high SNRs, the probability of a single bit error in a $U$-bit symbol is significantly higher, than that of two bit errors, assuming that the MRC outputs are i.i.d. random variables. Hence, the UUB probability of an intracode error in (13) can be approximated by

$$
P_{\text {intra }}(I I)=\sum_{j=2}^{U+1} Q\left(\sqrt{\frac{\left|X_{11}-X_{1 j}\right|^{2}}{4 \sigma_{N}^{2}}}\right)
$$

where we replaced $U p$ by $I I$, in order to indicate that this is now not the UUB of an intracode error according to Approach II. Furthermore, $X_{11}$ and $X_{1 j}, j=2,3, \ldots, U+1$, are two intracodes activating the same subcarriers, but having a distance of "one" between them, representing a one-bit error. Hence, the cross correlation between them is $(1-(2 / U))$, and consequently the error probability of (27) can be written as [19]

$$
P_{\text {intra }}(I I \mid S)=U Q(\sqrt{\bar{\gamma} S}) \text {. }
$$

By contrast, for an intercode error under sufficiently high SNRs, according to Fig. 3, the metrics computed from (12) for the specific data vectors $\mathbf{D}_{\mathbf{i}}$ other than the transmitted vector generate the maximum metric, if the bits of $\mathrm{D}_{\mathbf{i}}$ in the positions corresponding to the activated subcarriers of the transmitter were identical to the transmitted bits. These cases constitute the most probable events of intercode errors and hence (14) can be approximated by

$$
P_{\text {inter }}(I I)=2^{v}[A(Q, 2 v, U)-1] Q\left(\sqrt{\frac{\left|X_{11}-X_{i j}\right|^{2}}{4 \sigma_{N}^{2}}}\right)
$$

where, again, $U p$ was replaced by $I I$, in order to avoid confusion with the UUB of the intercode error probability, while $X_{11}$ and $X_{i j}$ are two intercodes having a minimum distance of $d=2 v$. The conditional probability of error in (29) can be expressed as

$$
P_{\text {inter }}(I I \mid S)=2^{v}[A(Q, 2 v, U)-1] Q\left(\sqrt{\frac{v \bar{\gamma} S}{2}}\right) .
$$

Note that in deriving (30), according to Fig. 3, the cross correlation between $X_{11}$ and $X_{i j}$ is $(1-(v / U))$, since the " 0 " elements (off-state) of the CWC are included in the correlation computations.

The total BER conditioned on the tap attenuations is constituted by both intra and intercode errors, which can be approximated as

$$
P(I I \mid S)=\frac{1}{U} P_{\text {intra }}(I I \mid S)+\frac{1}{2} P_{\text {inter }}(I I \mid S)
$$

according to the above analysis. The average BER for a given number of hits $K_{h}$ is calculated from the conditional bit-error probability of (31) by averaging it with respect to the pdf of $S$, $P(S)$, which is given by (19), yielding after integration

$$
\begin{aligned}
P^{I I}\left(K_{h}, \gamma_{s}\right) & =P\left(K_{h}, \gamma_{s}\right) \\
& +2^{v-1}[A(Q, 2 v, U)-1] P\left(K_{h}, \frac{v \gamma_{s}}{2}\right)
\end{aligned}
$$

where $P\left(K_{h}, x\right)$ is given by (21).

In conclusion, the average BER of the receiver using the blind soft-detection Approach II can be computed by substituting (26) and (32) into (25) with $P^{I}\left(K_{h}, \gamma_{s}\right)$ replaced by $P^{I I}\left(K_{h}, \gamma_{s}\right)$.

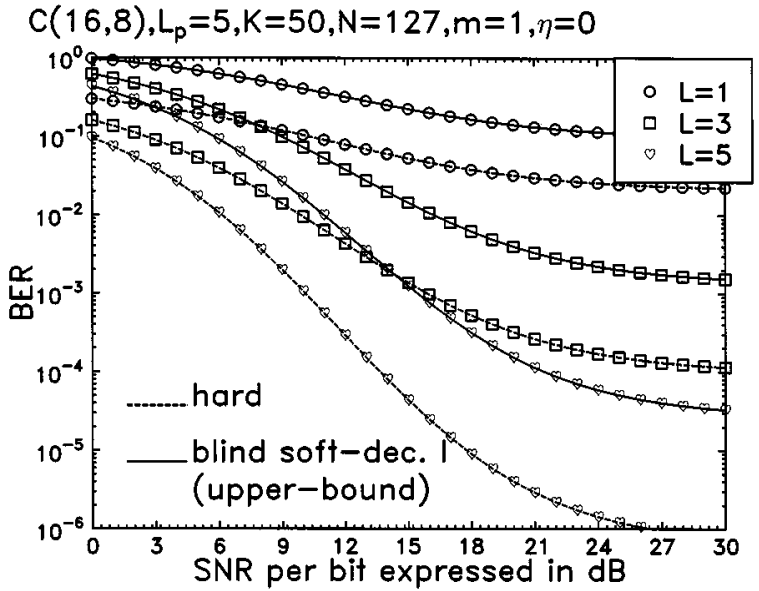

Fig. 4. Comparison of the BER versus bit-SNR performance between hard-detection and blind soft-detection using Approach I for $m=1$ evaluated from (24)-(26) for the $\mathrm{CWC} \mathrm{C}(16,8), L_{p}=5$ resolvable paths, diversity combining order $L=1,3,5, K=50$ users, bit-duration to chip-duration ratio of $N=127$ and MIP decay factor $\eta=0$. The corresponding parameters of the other figures are explicitly stated at the top of the illustrations. For $m=1$, the BER performance of hard-detection outperforms that of the blind joint soft-detection using Approach I.

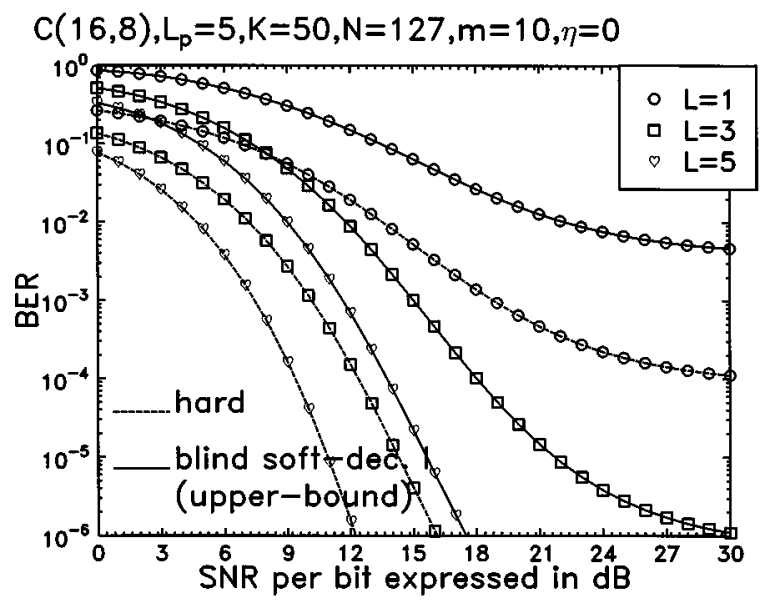

Fig. 5. Comparison of the BER versus bit-SNR performance between hard-detection and blind soft-detection using Approach I for $m=10$ evaluated from (24)-(26). For $m=10$, the BER performance of hard-detection outperforms that of the blind joint soft-detection using Approach I. BER decreases when increasing the value of $m$, i.e., fading is less severe compared with Fig. 4.

\section{NUMERICAL RESULTS}

In this section, the average BER performance is evaluated as a function of the average SNR per bit, which is obtained by computing

$$
\bar{\gamma}_{b}=\frac{\left(1-e^{-L_{p} \eta}\right)}{1-e^{-\eta}} \cdot\left(\frac{\Omega_{0} E_{b}}{N_{0}}\right)=q\left(L_{p}, \eta\right)\left(\frac{\Omega_{0} E_{b}}{N_{0}}\right)
$$

for all systems described above.

In Figs. 4 and 5, we estimated the upper-bound BER of Approach I upon combining $L=1,3$, and 5 paths in the receiver. In the figures, the BER of hard-detection was also plotted as a benchmarker for comparisons, upon assuming that the receiver exploited the explicit knowledge of the FH patterns. The parameters related to the computations were identical, as shown in 


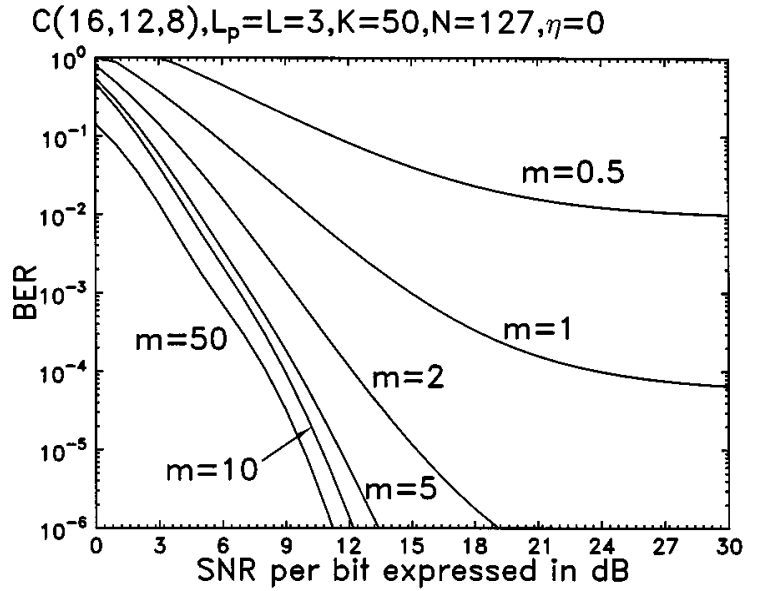

Fig. 6. BER versus SNR per bit performance of the CWC based SFH/MC DS-CDMA system using the blind soft-detection Approach II and fading parameters, $m$, in the range of $1 / 2$ to 50 evaluated from (25), (26), and (32). The system performance is critically affected by the communication environment encounted.

the figures, except that in Fig. 4, $m=1$ representing Rayleigh fading, while in Fig. 5, $m=10$ modeling Rician fading were used. The results show that in both of the above fading environments, the system provides dramatic BER improvements, when the number of combined diversity paths $L$ increases. However, the results also demonstrate that opting for the blind joint soft-detection Approach I increased the BER with respect to hard-detection. Furthermore, the results indicate that increasing the value of $m$ results in an improved blind detection BER performance for a given SNR per bit and for a given multipath diversity order of $L$. In Fig. 5, we found for $L=5$ that the SNR difference of the hard-detection and the blind detection Approach I is about $5 \mathrm{~dB}$ at the BER of $10^{-6}$.

Fig. 6 shows the BER performance for Approach II with respect to the multipath fading parameters $m=0.5,1,2,5,10,50$ upon evaluating (32), (26), and (25). The parameters used are shown in the figure. As expected, for a given SNR per bit, the BER decreases upon increasing the value of $m$, which implies that the fading becomes less severe. The system performance is critically affected by $m$, i.e., the communication environment encountered.

In Fig. 7, we evaluated the intracode word-error rate (WER) and the intercode WER, as well as their sum for the SFH/MC DS-CDMA system using the blind joint soft-detection Approach II, invoking the $\mathrm{FH}$ description code of $\mathrm{C}(16,12,8)$, i.e., using a minimum distance of $d=12$ between the CWCs, which corresponded to $v=6$. Since the intracode word-error probability is the codeword-error probability of the soft-detection, when the receiver has the a priori knowledge of the FH patterns, the results shown in this figure explicitly illustrate the comparison of the WER performance between the SFH/MC DS-CDMA system using blind joint soft-detection and that using soft-detection with a priori knowledge of the $\mathrm{FH}$ patterns. From the results, we concluded that the total WER was dominated by one of its contributing factors. Namely, for very low SNR per bit values, it was dominated by the WER of the intercode decisions, while for moderate to high SNRs per bit, by the WER of the intracode errors. Alternatively, from the

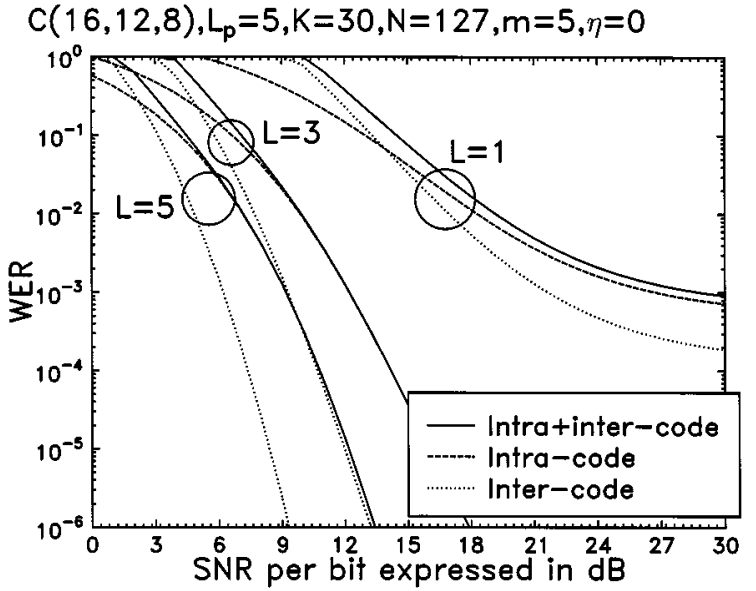

Fig. 7. WER versus SNR per bit performance of the CWC based SFH/MC DS-CDMA system using the blind soft-detection Approach II. For $v>2$ and sufficiently high SNR per bit, the average error probability of the blind joint soft-detection using Approach II is dominated by the intracode errors.

results and from the fact that the WER probability of the blind joint soft-detection is the sum of the intracode and intercode WER, the blind joint soft-detection should be outperformed by the soft-detection using the a priori knowledge of the FH patterns. This becomes explicit for $L=1$ and for $L=3,5$ at relatively low SNR per bit in Fig. 7. However, for $L=3,5$ at sufficiently high SNR per bit values, there is effectively no difference between the WER performance curves of the blind joint soft-detection (intra+intercode curve) and the soft-detection using the a priori knowledge of the FH patterns (intracode curve). Since the intercode WER is a function of $v$, hence, from (28) and (30), we infer that, if $v>2$, the total WER will be dominated by (28), provided that the SNR per bit is sufficiently high. This property suggests that for $v>2$, error-control techniques can be introduced, in order to correct the intracode errors by increasing the minimum distance between the intracodes, and hence to decrease the intracode WER of (28), consequently decreasing the total WER of the blind soft-detection Approach II.

Fig. 8 compared the performance of hard-detection upon exploiting the explicit knowledge of $\mathrm{FH}$ patterns to that of the blind joint soft-detection Approach II for different fading parameters, namely for $m=1,2,10$. The results show that for a sufficiently high SNR per bit and for a sufficiently good channel state - in contrast to the blind soft-detection Approach I-the BER performance of the blind soft-detection Approach II was superior to that of the hard-detection. This is because that in the blind soft-detection Approach II, the FH patterns invoked the CWCs having a minimum distance of $d=2 v$, and consequently, which improved the BER performance. Furthermore, if we can introduce error-control techniques reducing the intracode recognition error probability-as we discussed in the context of Fig. 7-we can further improve the BER performance of the blind soft-detection Approach II by decreasing the BER inflicted by intracode errors.

In our previous discussions in the context of Figs. 4-8, a constant information rate was assumed in each investigation. Under this assumption, for a bit duration of $T_{b}$, the duration of the transmitted signal was $U T_{b}$, if $U$ subcarriers were activated for 


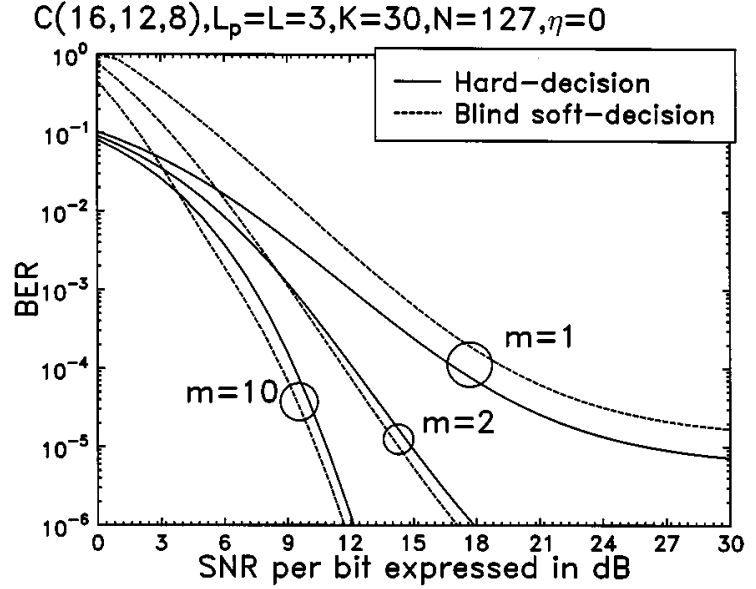

Fig. 8. BER versus SNR per bit performance comparison between the hard-detection and blind soft-detection for the CWC based SFH/MC DS-CDMA system evaluated from (25), (26), and (32). For sufficiently high SNR per bit and for a sufficiently good channel state, the BER performance of the blind joint soft-detection Approach II is superior to that of the hard-detection.

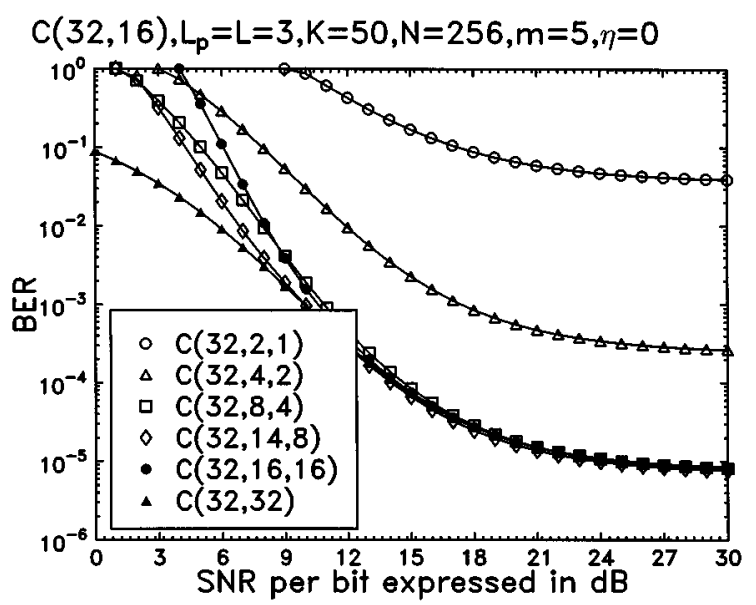

Fig. 9. BER versus SNR per bit performance of the CWC based SFH/MC DS-CDMA system using the blind soft-detection Approach II, under the assumption of constant spreading gain for multirate based systems evaluated from (25), (26), and (32). Under the multirate transmission, similar BER performance can be achieved by invoking limitations on the transmitted $\mathrm{FH}$ codes.

transmissions. Due to the large symbol duration in this system, the signal exhibits attractive anti-ISI characteristics. However, where multirate transmission is required, due to the constant symbol duration constraint a number of subcarriers have to be invoked, in order to support variable-rate operations. In this scenario, the processing gain is a constant.

Accordingly, in Fig. 9, a multirate communication system was characterized using (25), (26), and (32), under the assumption that all the interfering users employed the same CWC, namely $\mathrm{C}(32,16)$. Let the rate of each transmitted subcarrier be $R_{b}$. Then, the practical rates supported by the system might be $R_{b}, 2 R_{b}, 4 R_{b}, 8 R_{b}, 16 R_{b}$, or $32 R_{b}$, since the CWCs $\mathrm{C}(32,2,1)$, ..., $\mathrm{C}(32,16,16), \mathrm{C}(32,32)$ were assumed, as shown in the figure. By observing the curves associated with $\mathrm{C}(32,8,4)$, $\mathrm{C}(32,14,8), \mathrm{C}(32,16,16)$, and $\mathrm{C}(32,32)$, we conclude that even

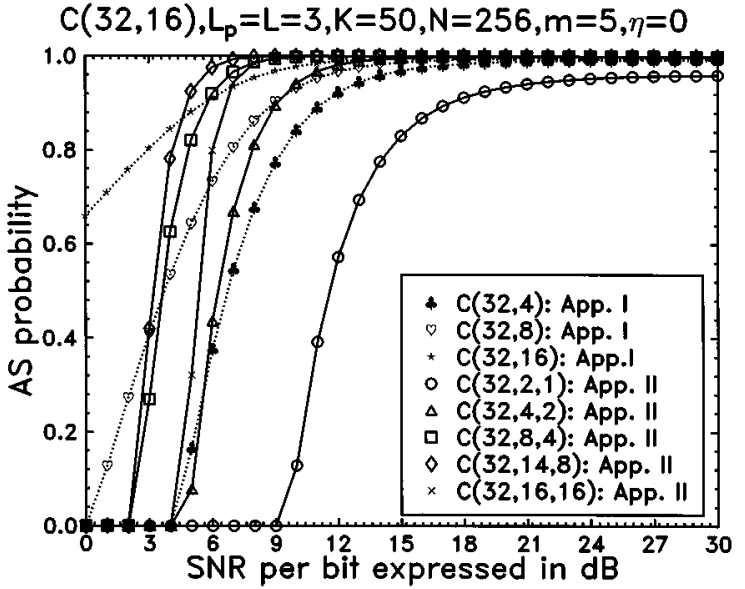

Fig. 10. Acquisition success (AS) probability performance of the CWC based SFH/MC DS-CDMA system using the blind soft-detection Approach I and II, under the assumption of constant spreading gain for multirate based systems. If the SNR per bit is sufficiently high, blind joint soft-detections can acquire the FH patterns used with high probability, while detecting the transmitted information.

though the systems transmit at different rates, a more or less similar BER performance can be maintained, when the channel quality is sufficiently high-assuming appropriate CWCs. By contrast, the BER performance of the system using the CWCs of $C(32,2,1)$ and $C(32,4,2)$ was inferior with respect to the others. The results can be explained by the help of (28), (30), and (31), where (31) is the weighted sum of (28) and (30). Due to the high minimum distance of the codes $\mathrm{C}(32,8,4)$, $\mathrm{C}(32,14,8), \mathrm{C}(32,16,16)$, and $\mathrm{C}(32,32)$, their associated BER performance is dominated by (28), while the BER of the systems using $C(32,2,1)$ and $C(32,4,2)$ is dominated by $(30)$, due to their relatively low minimum distance.

Finally, in Fig. 10, we characterized the AS probability of the blind soft-detection Approach I and that of the blind softdetection Approach II for the multirate transmission scenario discussed above, under the assumption that all the interfering users employed the same CWC, namely $\mathrm{C}(32,16)$. From the results, we observe that, for Approach I the AS probability becomes higher, when the CWCs associated with higher information rates are used, while for Approach II and for the special set of CWCs we used, $\mathrm{C}(32,14,8)$ achieved the best performance. Nevertheless, if the SNR per bit is sufficiently high, all the curves will reach an AS probability of approximately one.

This allows the receiver to blindly acquire a restricted set of FH patterns exhibiting a minimum distance of $d$, which can be used by the transmitter to signal the actual FH codes used for the transmission of "payload" information to the receiver. According to the above philosophy, a set of CWC codewords having a minimum distance of $d$ is used to convey the side information constituted by the FH codes to be used by the receiver for payload information recovery. During the consecutive information transmission, a randomly selected set of FH patterns from the $\left(\begin{array}{c}Q \\ U\end{array}\right)$ number of FH codes can be used. This does not impose any minimum distance limitations. In other words, following the blind detection of the "side information" constituted by the FH codes used by the transmitter, successive communications can be based on the explicit knowledge of the FH patterns. 


\section{CONCLUSIONS}

The proposed SFH/MC DS-CDMA system can efficiently amalgamate the techniques of SFH, OFDM, and DS-CDMA. Nonlinear CWCs have been introduced in order to control the associated FH patterns and, hence, to activate a number of subcarriers, in order to support multirate services. Blind joint soft-detection has been proposed for signal detection, when assuming no a priori knowledge of the FH patterns used. The properties of the constant-weight FH codes have been investigated in conjunction with specific system parameters, and the performance of the proposed SFH/MC DS-CDMA systems has been evaluated, under the conditions of supporting constant-rate or multirate services. From the results, we concluded that the proposed blind joint soft-detection techniques are capable of detecting the transmitted information and simultaneously acquiring the $\mathrm{FH}$ patterns. Our future work in this field will include multiuser detection, adaptive detection, and multiuser interference cancellation, in order to reduce the multiple-access interference. Fast FH and adaptive FH techniques will be invoked, in order to mitigate the effects of different fading channels and modulation schemes other than BPSK, such as various burst-by-burst adaptive quadrature amplitude modulation will be studied.

\section{ACKNOWLEDGMENT}

The authors would like to thank the anonymous reviewers for their constructive critique of the paper.

\section{REFERENCES}

[1] D. N. Knisely, S. Kumar, S. Laha, and S. Nanda, "Evolution of wireless data services: IS-95 to CDMA 2000," IEEE Commun. Mag., vol. 36, pp. 140-149, Oct. 1998.

[2] E. Dahlman, B. Gudmundson, M. Nilsson, and J. Skold, "UMTS and IMT-2000 based on wideband CDMA," IEEE Commun. Mag., vol. 36, pp. 70-80, Sept. 1998.

[3] E. A. Sourour and M. Nakagawa, "Performance of orthogonal multicarrier CDMA in a multipath fading channel," IEEE Trans. Commun., vol. 44, pp. 356-367, Mar. 1996.

[4] Y. Sanada and M. Nakagawa, "A multiuser interference cancellation technique utilizing convolutional codes and orthogonal multicarrier modulation for wireless indoor communications," IEEE J. Select. Areas Commun., vol. 14, pp. 1500-1509, Oct. 1996.

[5] Q. Chen, E. S. Sousa, and S. Pasupathy, "Multicarrier CDMA with adaptive frequency hopping for mobile radio systems," IEEE J. Select. Areas Commun., vol. 14, pp. 1852-1858, Dec. 1996.

[6] S. Kondo and L. B. Milstein, "Performance of multicarrier DS CDMA systems," IEEE Trans. Commun., vol. 44, pp. 238-246, Feb. 1996.

[7] L. Vandendorpe, "Multitone spread spectrum multiple access communications system in a multipath Rician fading channel," IEEE Trans. Veh. Technol., vol. 44, pp. 327-337, May 1995.

[8] P. Jung, F. Berens, and J. Plechinger, "A generalized view on multicarrier CDMA mobile radio systems with joint detection: Part I," Frequenz, vol. 51, no. 7/8, pp. 174-185, July/Aug. 1997.

[9] L. L. Yang and L. Hanzo, "Slow frquency-hopping multicarrier DS-CDMA," in Proc. Int. Symp. Wireless Personal Multimedia Communications (WPMC'99), Amsterdam, The Netherlands, Sept. 21-23, 1999, pp. 224-229.

[10] - "Blind soft-detection assisted frequency-hopping multicarrier DS-CDMA systems," in Proc. IEEE GLOBECOM'99, Rio de Janeiro, Brazil, Dec. 5-9, 1999, pp. 842-846.

[11] N. Nakagami, "The $m$-distribution, a general formula for intensity distribution of rapid fading," in Statistical Methods in Radio Wave Propagation, W. G. Hoffman, Ed. Oxford, U.K.: Pergamon, 1960.
[12] M. K. Simon and M. Alouini, "A unified approach to the probability of error for noncoherent and differentially coherent modulations over generalized fading channels," IEEE Trans. Commun., vol. 45, pp. 1625-1638, Dec. 1998.

[13] T. Eng and L. B. Milstein, "Coherent DS-CDMA performance in Nakagami multipath fading," IEEE Trans. Commun., vol. 43, pp. 1134-1143, Feb./Mar./Apr. 1995.

[14] L. L. Yang and C. S. Li, "DS-CDMA performance of random orthogonal codes over Nakagami multipath fading channels," in Proc. 4th IEEE ISSSTA'96, Mainz, Germany, Sept. 22-25, 1996, pp. 68-72.

[15] G. P. Efthymoglou, V. A. Aalo, and H. Helmken, "Performance analysis of coherent DS-CDMA systems in a Nakagami fading channel with arbitrary parameters," IEEE Trans. Veh. Technol., vol. 46, pp. 289-296, May 1997.

[16] _ - "Performance analysis of noncoherent DS-CDMA systems in a Nakagami fading channel with arbitrary parameters," IEE Proc. Commun., vol. 144, no. 3, pp. 166-172, June 1997.

[17] T. Vlachos and E. Geraniotis, "Performance study of hybrid spread-spectrum random-access communications," IEEE Trans. Commun., vol. 39, pp. 975-985, June 1991.

[18] J. Wang and M. Moeneclaey, "Hybrid DS/SFH-SSMA with predetection diversity and coding over indoor radio multipath Rician-fading channels," IEEE Trans. Commun., vol. 40, pp. 1654-1662, Oct. 1992.

[19] J. G. Proakis, Digital Communications, 3rd ed. New York: McGrawHill, 1995.

[20] S. Verdu, Multiuser Detection. Cambridge, U.K.: Cambridge Univ. Press, 1998.

[21] S. M. Johnson, "A new upper bound for error-correcting codes," IRE Trans. Inform. Theory, vol. IT-8, no. 2, pp. 203-207, 1962.

[22] F. J. Macwilliams and N. J. A. Sloane, The Theory of Error-Correcting Codes. New York: North-Holland, 1977.

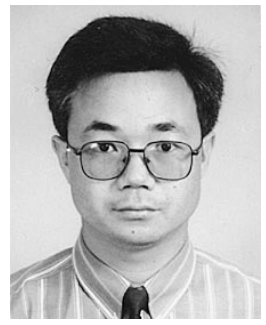

Lie-Liang Yang (M'98) received the B.Eng. degree in communication engineering from Shanghai TieDao University, Shanghai, China, in 1988, and the M.S. and Ph.D. degrees in communications and electronics from Northern Jiaotong University, Beijing, China, in 1991 and 1997, respectively.

From 1991 to 1993, he was a Lecturer with the Department of Electrical Engineering, East-China Jiaotong University, China. From 1993 to 1997, he was with the Modern Communications Research Institute, Northern Jiaotong University, China. From June 1997 to December 1997, he was a Visiting Scientist of the Institute of Radio Engineering and Electronics, Academy of Sciences of the Czech Republic. Since December 1997, he has been with the Communication Group, Department of Electronics and Computer Science, University of Southampton, U.K., and is involved in researching various error correction coding, modulation, synchronization, and spread spectrum systems for future generations of wireless mobile communication systems. He has published over 40 papers in journals and conference proceedings

Dr. Yang was awarded the Royal Society Sino-British Fellowship in 1997.

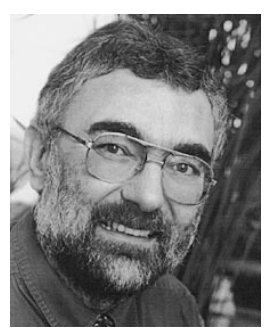

Lajos Hanzo (M'91-SM'92) graduated in electronics in 1976, and in 1983, he received the Ph.D. degree.

During his 24-year career in telecommunications, he has held various research and academic posts in Hungary, Germany, and the U.K. Since 1986, he has been with the Department of Electronics and Computer Science, University of Southampton, U.K., and has been a Consultant to Multiple Access Communications Ltd., U.K. Currently, he holds the Chair in Telecommunications. He co-authored five books on mobile radio communications, published about 300 research papers, organized and chaired conference sessions, presented overview lectures, and was awarded a number of distinctions. Currently, he is managing a research team, working on a range of research projects in the field of wireless multimedia communications under the auspices of the Engineering and Physical Sciences Research Council (EPSRC) UK, the European IST Programme, and the Mobile Virtual Centre of Excellence (VCE). He also provides a range industrial training courses. For further information on research in progress and associated publications, please see the website at http://www-mobile.ecs.soton.ac.uk. 